\title{
Journal of Neurodegenerative Disorders
}

ISSN: $2642-4274$

Research Article

DOI: $10.36959 / 459 / 606$

\section{The Experience of Parkinson's Disease in Hai District, Tanzania}

\author{
Natasha Fothergill-Misbah ${ }^{1 *}$, Catherine Dotchin ${ }^{1}$, John Kisima ${ }^{2}$, Kate Hampshire ${ }^{3}$, and Richard Walker ${ }^{1}$ \\ ${ }^{1}$ Population Health Sciences Institute, Newcastle University, UK \\ ${ }^{2}$ Hai District Hospital, Tanzania \\ ${ }^{3}$ Department of Anthropology, Durham University, UK

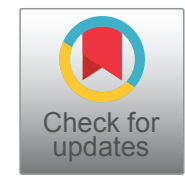

\begin{abstract}
Background: Parkinson's disease (PD) is a debilitating neurological disease that can result in a reduced quality of life for people with Parkinson's disease (PwP) and their families. The incidence of PD continues to increase as the global population ages, as is being experienced in Tanzania and much of sub-Saharan Africa, yet healthcare systems are under-developed and not prepared to manage the burden posed by chronic conditions. Little is known about people's experiences of long-term drug treatment for PD and the impact disease progression has on PwP and their families' lives. This paper outlines findings from a small-scale qualitative research study on the experiences of PwP in later stages of illness and their families in the Hai district of Tanzania.
\end{abstract}

Methods: Semi-structured biographical in-depth interviews were conducted with three PwP and six caregivers in the Hai district of Tanzania. Interviews explored participants' experiences of living with PD and caring for a family member with $\mathrm{PD}$, their response to biomedical treatment, use of alternative treatment practices and understanding about the disease.

Results: PWP and caregivers all expressed the emotional, social, physical and economic strain of PD. PwP felt angry and frustrated with their deteriorating condition and increasing dependence but pleased with the treatment. Caregivers described the full-on nature of their caring role which resulted in increasing social isolation, worry and stress as the PwPs' condition progressed. Religion, faith and prayers played a significant role in disease management in combination with pharmaceutical treatment. Participants did not report any stigmatisation towards PwP from communities.

\begin{abstract}
Conclusion: This study outlines the emotional, financial and physical challenges that PwP at advanced disease stages and their caregivers experienced in the Hai district. It highlights the need for PD management to acknowledge the role of spiritual healing and social support in combination with biomedical treatment to achieve effective care and improve the wellbeing of PWP and their families, and the need for increased awareness and understanding about PD in the region.
\end{abstract}

\section{Keywords}

Parkinson's disease, Experiences, Management, Tanzania, Sub-saharan Africa

\section{Abbreviations}

PD: Parkinson's Disease; PWP: People with Parkinson's Disease; SSA: Sub-Saharan Africa, DSS: Demographic Surveillance Site; NHS: National Health Service

\section{Background}

Parkinson's disease (PD) is the second most common neurodegenerative disorder globally [1], characterised by tremor, muscle rigidity, bradykinesia (slowness of movement), postural instability [2] and an array of non-motor symptoms. $P D$ increases in prevalence with age. Estimates for the prevalence of PD globally have varied, which is thought to be due to a difference in access to diagnosis and awareness among people and healthcare professionals [3]. A door-to-door prevalence study conducted in the Hai demographic surveillance site (DSS) in northern Tanzania demonstrated a higher prev-
*Corresponding author: Natasha Fothergill-Misbah, Population Health Sciences Institute, Newcastle University, Newcastle upon Tyne, Baddiley Clark Building, NE1 7RU, UK

Accepted: February 13, 2021

Published online: February 15, 2021

Citation: Fothergill-Misbah N, Dotchin C, Kisima J, et al. (2021) The Experience of Parkinson's Disease in Hai District, Tanzania. J Neurodegener Disord 4(1):122-128 
alence of PD $(40$ per 100,000$)$ than that previously demonstrated in sub-Saharan Africa (SSA) [4].

Poor awareness about PD in SSA $[5,6]$ and limited access to services results in people with PD (PwP) often not obtaining a diagnosis [7] or not receiving medication because of poor availability and affordability of drug treatment [8-10]. Symptomatic drug therapy with Dopaminergic drugs is effective in improving motor symptoms [11] but does not alter the course of the disease; symptoms will progress and impact on the wellbeing of PwP and caregivers [12-14]. Lack of public social care and health services in rural Tanzania result in the main burden of care being met by families [15-17]. However, increasing urban migration, economic strain and poverty are thought to be contributing to an isolated, vulnerable ageing population $[18,19]$.

Mshana, et al. [3] conducted a qualitative study in the Hai DSS in Tanzania to identify the experiences and treatment-seeking behaviours of PWP before, and shortly following, diagnosis. PwP from this study expressed bitterness about the symptoms and economic impact on their family [3], even at early stages of disease, while caregivers felt socially isolated and helpless. More recently in Ethiopia, Walga [20] described the difficulties caring for PwP, limited understanding of PD and limited support for caregivers.

To develop culturally appropriate chronic disease policies, it is necessary to understand people's experience of chronic illness and the social, economic and political factors influencing their care and use of health services [21] and alternative healing [22]. This study aims to explore the experiences of PWP who have been living with PD for at least 6 years to understand how disease management could be improved. The study explores how PwP and families manage late stage PD in this resource poor context with very little information about the disease, no formal social support and limited access to biomedical services or specialists.

\section{Methods}

\section{Study site and population}

The study was conducted within the Hai DSS in North-eastern Tanzania with an area of approximately $2,000 \mathrm{~km}^{2}$. The majority of the population of Hai are from the largest ethnic group in the region, Chagga (75\%), with also Maasai and other ethnic groups [3]. Christians and Muslims constitute the vast majority of the population. All participants resided in the fertile mountainous terrain of the district in the foothills of Mt. Kilimanjaro, and in the less fertile plains below, with agriculture, livestock keeping and farming of coffee and bananas being the main source of income for families [23].

\section{Study participants and procedures}

PWP included in this study were identified from two studies within the Hai DSS. These included a door-to-door community-based prevalence study conducted in 2006 [4] and a neurological disability prevalence study in 2010 [24]. Dotchin, et al. [4] identified 32 PwP in 2006, while Dewhurst, et al. [24] identified 13 PwP in 2010. A total of 20 PwP were alive at the time of this study in 2016.
A convenience sample of PwP and main caregivers were invited to take part in-depth semi-structured interviews. Convenience sampling was used as of the 20 PwP who were still alive from the prevalence studies in 2006 and 2010, five were too cognitively impaired to consent to participation, six were difficult to locate, their villages challenging to access or they did not consent to participation, owing to the small sample size. All PwP $(n=9)$ identified in the Hai DSS who were accessible, and willing and able to participate were seen. Enumerators from each village were approached to assist with locating the participants. Main caregivers were identified as those people who provided the majority of care for a PwP. Participants all spoke Kiswahili, the national language, but the majority did not speak English. Participants generally had few resources; most owned radios but not televisions, most had access to newspapers in the local villages but no access to libraries or the internet. The main source of income for participants was small-scale farming and/or family run businesses (i.e. village shop). All but one of the participants received PD treatment for free and was followed up annually. The participant had not received treatment since diagnosis as she could not be located by the medical officer supplying medication; the research team located her with the help of a local village enumerator and thus supplied her with medication.

PwP identified had lived with PD for at least six years, and many had suffered for more than 10 years (since being identified in 2006). For the majority, their condition had deteriorated significantly. If PwP were too unwell to interview (i.e. bedbound) or their speech was impaired, PwP were represented by their caregiver who was interviewed on their behalf, with their permission and consent. Interviews represented nine PwP, with three PwP directly interviewed and six caregivers interviewed on behalf of PwP. All participants had given consent to take part in a previous study and were aware of the procedures. Interviews lasting 45-90 minutes were conducted in the participants' own homes in Kiswahili with an interpreter who translated verbatim, and were audio recorded with consent. Demographic questions included disease, treatment and caregiver duration, caregiver relationship, age and religion. Interviews followed a biographical approach, and explored life before PD, experience of biomedical treatment, use of alternative treatment, knowledge about PD, experience of disease progression and symptoms and caregivers' experiences of the caring role.

Participants were given written and verbal information (in Kiswahili) about the purpose of the study and made aware their participation was entirely voluntary and they had the ability to withdraw at any time or decline to answer any questions. Confidentiality and anonymity were ensured prior to commencing interviews and consent was obtained through a signature $(n=8)$ or thumb print $(n=1)$. PwP gave consent for their caregiver to be interviewed on their behalf, and verbal consent to record the interviews was obtained prior to starting the interviews. Ethical clearance to conduct this study was obtained from National Institute for Medical Research and NHS Research and Ethics Committee, UK and permission was given by the Hai District Medical Officer to carry out the study. 


\section{Data Analysis}

Thematic analysis of interviews was carried out using an inductive approach, where analysis is data-driven [25]. The research followed Braun and Clarke's six-phase process of thematic analysis. Phase I involved familiarisation with the data; recordings were listened to in a reflexive manner to focus data collection and initial notes made. On leaving the field, recordings were transcribed verbatim into English from Kiswahili by a bilingual researcher. Phase II involved the generation of initial codes; notes and comments were made and supporting quotes identified from transcripts. Phase III-V involved searching, reviewing, defining and naming of themes; initial empirical and theoretical codes and supporting data were collated into themes to address the study's aims. Themes were reviewed, refined and defined by the team. Phase VI involved producing the report while ensuring that individual experiences and contexts emerged from the writing.

\section{Results}

The Kiswahili term for PD is 'ugonjwa wa kutetemeka', which translates as 'the shaking illness'. This is the term participants used to refer to PD.

\section{Demographics}

The reported age range of PwP was 60-101 years, of who three were female and six male. Exact disease duration prior to diagnosis was unknown by participants, although in all cases it was more than 6-years. One PwP had never taken treatment. All PwP had identified main caregivers, whose ages ranged from 18-64. All but two caregivers were present when conducting the interviews. The relationships of caregivers to PwP included wife $(n=3)$, daughter $(n=2)$, son $(n=2)$, daughter in law $(n=1)$ and granddaughter $(n=1)$. Duration of care ranged from 2-12 years. Eight participants identified as Christian, and one Muslim.

\section{Experiences of PWP}

All participants identified that PwPs' conditions had deteriorated over time, despite taking symptomatic treatment. However, most PwP and caregivers expressed their joy that medication relieved their symptoms, allowed PwP to carry out daily tasks of living again, socialise and continue working in some cases. PwP were happy they had regained some independence, even if it was only small feats such as getting themselves out of bed, standing up, feeding themselves or walking to their farm or friend's home. These achievements allowed them to have more control over their lives than they previously had:

"She could walk to the neighbours and come back by herself, she can take bath by herself, she goes to the toilet alone, uses a stick to walk and she is not falling anymore".

\section{Ms O's daughter, 18-years}

Although medication allowed several PwP to live more independent lives, other participants described the impact of PwPs' deteriorating condition. Many PwP could no longer perform acts of daily living, which resulted in them becoming withdrawn from their communities and caregivers taking on additional tasks:

"She can't do anything now, all that she used to do by herself now she needs assistance to do... [She is] unable to walk, do her own stuff like teeth brushing or showering...She just couldn't do anything to help herself when this condition appeared".

\section{Ms R's daughter, 64-years}

In addition to the physical challenges PwP experienced, two also experienced memory loss. Mr S. had no one to care for him during the day, he seemed extremely forgetful during the interview, acknowledging that he found it difficult to remember things. Consequently, he would often forget to take his medication or would take additional doses during the day, resulting in sub-optimal treatment and side effects such as dizziness. Apathy and lack of motivation was also an issue for several PwP, one 75-year-old PwP refused to eat, drink or take his medication:

"Since six months ago when he lost his memory he stopped taking the drugs... The drugs were working very well but he refuses to take them, we think because of his memory".

\section{Mr N's son, 43-years}

Several PwP also described the emotional impact of their worsening symptoms, their anger regarding their increasing dependence on others for care and sadness at being ill. PwP recalled the things they could do in the past and thought their lives had changed for the worse; pervasive low mood was common.

"I felt very bad, I did not like it, I was angry at myself because I couldn't wake up, I would take hours to get out of bed".

Mr Q, 92-years

Feelings expressed by PwP included frustration, isolation, restriction, bitterness, hopelessness, incapability and being a burden. For some, the symptoms of PD meant PwP were unable to uphold social expectations, such as standing up to greet someone when they visited, or offering a friend or child a gift, demonstrating the impact of PD on sociality.

\section{Experiences of caregivers}

The majority of caregivers talked of their unhappiness that their loved ones were ill, but also of how they had lost the ability to lead their own lives. One daughter described her role caring for her mother as "kila kitu, kila siku, kila saa", which translates as "everything, every day, every hour". Caregivers described the increasing roles they took on as the symptoms of PD developed over the years, including cooking, washing up, feeding, dressing, carrying PWP and providing their treatment among other tasks. However, some noted how commencing treatment had improved PwPs' condition and allowed caregivers to leave their side at times.

Full-time caregivers in particular described the significant impact PD had on their lives, where care giving was a full-time job and resulted in physical and emotional strain: 
"I get tired because it is only me both during the day and night. I carry her, dress her, wash and cook for her. You can't do any other work".

\section{Ms R's daughter, 64-years}

Full-time caring roles also resulted in caregivers feeling more isolated and under more economic strain. Several described how they had left their previous 'lives' and jobs to care for their relative with PD. They also described not having time to do activities they used to, such as going to the market, seeing friends or going to church:

"I have nothing, I can't leave her alone. She might need my help any time. I have no one to take care of her if I leave".

\section{Ms R's daughter, 64-years}

Caregivers who were unable to leave the house described feeling socially isolated, stressed, constantly worried and depressed on occasions. However, they had not sought professional help, praying instead. In contrast, caregivers who had extensive families and shared the caring role with others described the strong support network they had around them, as well as their increased sources of income.

Some caregivers described the difficulty coming to terms with their changing roles. For example, Mr J's wife explained how hard it was to accept that she was now her husband's full-time caregiver rather than his wife, which she found stressful and upsetting. However, she added that he would not let anyone else care for him.

In addition to the emotional and physical challenges of carework, almost all caregivers described the increased financial difficulties they faced. Both PwP and full-time caregivers could not engage in paid work, while costly trips to hospitals because of falls or other comorbidities added up.

"The family has been suffering difficult times. We can't work at the farm...and we can't even go to the market because we can't leave him alone".

\section{Mr L's daughter, 49-years}

PD medication is provided free of charge to all PwP who were identified in the prevalence studies, but many expressed their concern about the drug supply being stopped and the fear of having to source medication themselves, not being able to afford it or having to sell assets. The daughter of one PwP described how they were completely dependent on donations from their community. However, even larger families with more resources described the financial implications of PD. Furthermore, PwP with fewer care opportunities described the dilemma they had between needing a caregiver but needing to maintain an income. For example, Mr S's memory loss meant that he needed someone to look after him. However, when asked about this he responded, "How can someone stay here without doing any work, how shall we live then". His wife, who was also his caregiver, had to continue working through the day to support them.

\section{PD management and cause of disease}

Almost all participants described how much of an im- provement they had seen in their symptoms after commencing treatment. However, participants also described the importance of their religious faith as well as family support networks in the management of PD. All participants described the role of faith and prayers in helping them come to terms, or deal, with their condition or caring role. Participants described praying every day and thanking God for what they had, for example: "I left everything to the Lord Jesus Christ". Participants believed both their medication and prayers were key to the management of PD:

"I think I have to take both the drugs and the prayers. So, I cannot know which one works".

Mr P, 60-years

Faith and drug treatment were complementary. Furthermore, all but one participant reported visiting faith healers (both Christian and Muslim) who performed spiritual rituals as well as prayers. Those who could not travel were visited at their homes weekly.

Only one participant described using herbal medicine, involving herbal leaves to wash the body. However, they reported it was not successful. Despite four participants suggesting that evil spirits or curses had caused PD, all participants expressed that seeking traditional healers went against their religion and therefore was not allowed.

In addition to evil spirits, participants had various suggestions regarding the cause of PD, which included: God $(n=3)$, old age $(n=3)$, work $(n=2)$, just a disease $(n=2)$, the rain ( $=1$ ) and snake venom $(n=1)$. Three participants identified that their community believed that PwP had been cursed or bewitched:

"Some said she is bewitched, some said they have sent her a devil...Some say because she is old, that is why the disease came to her. Some say she is not old enough to get that sick".

\section{Ms O's daughter-in-law, 18-years}

Despite these beliefs, participants reported that PwP were not stigmatised by their communities. Rather, friends and the community were supportive and often visited or prayed for them. Some participants described that after witnessing the improvement from pharmaceutical treatment, they believed that PD could be a medical disease.

\section{Discussion}

This study has followed up the same cohort of PwP and caregivers interviewed by Mshana, et al. [3] and other more recently diagnosed PwP [24]. Mshana, et al. conducted the only published social science study to date exploring PwP and caregivers' experiences of PD in SSA before and shortly following diagnosis. However, there are no data regarding PwPs' experiences of long-term drug treatment and the impact of PD progression on their lives.

This small-scale study has identified the social, physical, emotional and financial impact of advanced PD on both PwP and their caregivers. PwP experienced increasing dependence on others for activities of daily living, where they had to come to terms with a changing 'self'. Mshana, et al. [3] 
identified similar findings in their study, using Charmaz's 'loss of self' [26] to make sense of these experiences. Bramley and Eatough [27] identified similar findings in the UK where PD disrupted PwPs' sense of self. Among PwP in Hai, loss of independence also contributed to feelings of sadness and anger and social isolation, where they could not contribute to, or participate in, society. The importance of being able to maintain some form of independence became clear from PwPs' joy of being able to walk to a neighbour's house or go to the toilet by themselves, which their medication enabled them to do. This illustrates the importance of pharmaceutical treatment in allowing PwP to remain as independent as possible by delaying symptom progression. Dotchin, et al. [28] propose that early diagnosis and treatment of PD could also reduce the burden of care on the family.

Social support systems in rural Tanzania are lacking, with under-resourced health facilities and under-trained health professionals in relation to the management of chronic illness $[7,29]$. Support mechanisms for PwP in high-income countries include the use of therapeutic drugs, physiotherapy, speech and language therapy, occupational therapy, informal and formal care, information, resources and support groups [30]. In contrast, the full burden of care in Hai falls on informal family caregivers who often gave up their own lives to care for PwP with no additional support or information. Although family support has been linked to improved outcomes in people suffering from chronic disease [31], this can lead to increased stress, emotional strain and isolation among caregivers, as identified in Hai and among caregivers globally [27,32-35]. In high-income countries, support groups can play a beneficial role in enabling sociality [36] and as sources of information; further research would need to identify the benefits in the context of SSA.

Furthermore, caregivers' roles increased as PD symptoms progressed. Worsening symptoms also affected both PWP and caregivers' ability to greet friends, attend church, visit markets or neighbours, highlighting the stark social consequences of living with PD. Dotchin, et al. [28] conducted a study on the caregiver burden of PD and dementia in Hai using the Zarit Burden Interview (ZBI). They identified that caring for someone with PD was more burdensome than caring for someone with dementia, while advanced PD had a higher care burden.

Social support from extended families appeared to be an important way for caregivers to relieve the burden of their caring role. Care work was shared while families had increased income generating opportunities. In contrast, those without large families had no social, financial or emotional support. The family plays a crucial role in care for older people in SSA, especially where formal support is lacking [37]. However, literature has reported a decline in the material support available for older people due to economic constraints and increased urban migration $[38,39]$. Although larger families had more income generating opportunities, all participants expressed the financial burden of care. All PWP (bar one, as described) in this study were provided with treatment at no cost, so the impact of the cost of medication on households cannot be evaluated. However, it became clear that no families would be able to afford medications due to their high cost and poor availability. The financial implications of PD could be devastating for families who must source PD medication themselves. If families cannot afford or access treatment, PWP could experience significant suffering if their symptoms progress untreated, resulting in increased caregiver burden. As identified in both Tanzania and Kenya, PD medication is unaffordable for many and often unavailable in pharmacies, particularly in rural areas $[7,8]$.

Concerning the management of PD, participants stressed the importance of pharmaceutical medication but also the crucial role of faith healing and prayers. Faith provided a way for families to come to terms with, or make sense of, PD, allowed them to keep going and deal with their emotional stress and worry. Mshana, et al. [3] identified similar findings among PwP in Hai who sought out faith healing. Asare and Danquah [40] in their narrative review of the 'African Belief System' in Ghana acknowledge that for many Africans, wellbeing goes beyond healthcare and lifestyle and comprises spiritual involvement. However, just as within biomedicine, diversity in traditional, complementary and alternative medicine exists in different contexts, sub-cultures and for different health conditions. Mokgobi [41] suggests that societies develop their own ways of dealing with illness by drawing on "cultural relativism", where cultural context is "critical to an understanding of people's values, beliefs and practices" [42]. Omonzejele [43] in Nigeria and Mokgobi [41] in South Africa have recognised the importance of spirituality and faith in chronic disease management. Janzen [44] adds that in Western Equatorial Africa, "divination" is involved when dealing with misfortune or illness. Spirituality, faith, prayers and beliefs are vast and complex, yet it is clear that they play an important role in the management of illness, particularly those that biomedicine cannot 'cure', as experienced by participants in Hai and as reported elsewhere in SSA. It may be important in the future for spiritual beliefs to be considered within treatment programmes, while also educating spiritual healers on basic medical conditions. In the USA, Bingham and Habermann [45] found that spiritual beliefs gave meaning, hope and courage to PwP and caregivers to face PD. Furthermore, Soundy, et al. [36] in their systematic review and meta-ethnography identified the importance of spiritual strategies in healing and for maintaining hope among PwP.

Medical pluralism has been described as the 'reality' of African healthcare [46] where people seek out a range of treatment options. Although all participants described the importance of prayers, faith healing and biomedicine, it was unexpected that only one family reported trying herbal medicine. Traditional healing takes on many different forms and studies in SSA have described the reliance on traditional or herbal medicine for PD and other chronic conditions $[3,47,48]$. However, families suggested that traditional healing went against their religion and it was a sin to believe in witchcraft. Hampshire and Owusu [49] have described how churches in Ghana have condemned traditional medicine as it opposes their mission, while in Zambia, Sugishita [50] identified that witchcraft violated Christian values. There is also the possibility of selection bias where those who would engage with traditional healing may not have participated in the study or could have 
been less willing to participate. The sample size of this study was small, and so, may not have included those who do interact with alternative medicine. However, Marsland [51] and McMillen [52] have also discussed how "witchdoctors" in Tanzania could be remarketing themselves as "herbalists", adapting and improvising their practices to appeal to wider audiences and gain legitimacy. The boundaries between "herbal medicine", "traditional medicine" and "witchdoctors" are becoming increasingly blurred and may have contributed to these families not engaging with alternative therapies.

Finally, no participants reported feeling stigmatised because of their condition. The stigma associated with PD has been documented globally [53] and PD has previously been described as a 'problem of shame' in Tanzania [3]. PwP may have indeed experienced stigmatising perceptions initially, as Mshana, et al. identified, and this could have decreased through PwPs' positive responses to medication and the understanding of PD as a non-spiritual condition. However, it is also important to consider that most interviews were done with caregivers, which could lead to bias in perceptions; PwP may have experienced stigma but their views were not heard. Additionally, there could be a social pressure which leads PwP to say they had not been stigmatised, even if, in fact, they had. PD can contribute to social exclusion, as described by participants, and so PwP may not have been engaging with the community or putting themselves in situations where they could be judged.

\section{Limitations}

One of the main limitations to this study was the small number of people interviewed, and the fact that the interview was with the caregiver in six out of the nine cases. However, as noted, several PwP had died, were too cognitively impaired, inaccessible or did not give consent. Also, PwP were in late stage disease due to their relatively long disease duration of at least six years, and much longer in some. However, this allowed us to look at the impact of more complex symptoms such as cognitive impairment and the major impact these had on both PwP and caregivers. All but one PwP had free access to drug treatment so their views are not representative of those who do not, the majority of PwP in SSA. The study was carried out in one area of Tanzania so the results cannot be directly extrapolated to other areas of Tanzania and SSA. However, this is the first study to look at the views of PwP on long term treatment, and the impact on caregivers, in SSA and provides some very useful insights. We recommend future studies consider the experience of PD in other districts in Kilimanjaro, Tanzania and across wider SSA.

\section{Conclusion}

This study explored the experience of PD among PwP and caregivers living in a rural area of Tanzania. Much of the guidance of how to live well with PD comes from high-income countries and does not consider how sociocultural, and economic, factors influence disease experience. This study has demonstrated the significant emotional, physical and financial implications of PD in the context of Hai. Findings also illustrated the crucial role of the family in care for PwP as well as the significant social consequences of living with PD and being a full-time caregiver with no formal social support. The burden of care could be substantially reduced if PD was diagnosed and treated earlier, and health services that utilised the full spectrum of PD management, including multidisciplinary teams, were made available. However, this study has also identified the crucial role of faith and spiritual healing in the management of PD. These beliefs are integral to care and cannot be ignored. Findings have demonstrated the need for faith healers and biomedical healthcare professionals in Hai, and possibly other rural areas of Tanzania and SSA, to work together to manage PD and give hope, support and encouragement to PwP and families in order to improve their quality of life.

\section{References}

1. Connolly BS, Lang AE (2014) Pharmacological treatment of Parkinson disease: A review. Jama 311: 1670-1683.

2. DeMaagd G, Philip A (2015) Parkinson's disease and its management: Part 1: Disease entity, risk factors, pathophysiology, clinical presentation, and diagnosis. P T 40: 504-532.

3. Mshana G, Dotchin CL, Walker RW (2011) 'We call it the shaking illness': Perceptions and experiences of Parkinson's disease in rural northern Tanzania. BMC public health 11: 219.

4. Dotchin C, Msuya O, Kissima J, et al. (2008) The prevalence of Parkinson's disease in rural tanzania. Mov Disord 23: 1567-1672.

5. Kaddumukasa M, Kakooza A, Kaddumukasa MN, et al. (2015) Knowledge and attitudes of Parkinson's disease in rural and urban mukono district, uganda: A cross-sectional, community-based study. Parkinsons Dis 2015: 196150.

6. Mokaya J, Gray WK, Carr J (2017) Beliefs, knowledge and attitudes towards Parkinson's disease among a Xhosa speaking black population in South Africa: A cross-sectional study. Parkinsonism relat disord 41: 51-57.

7. Dotchin CL, Msuya O, Walker RW (2007) The challenge of Parkinson's disease management in Africa. Age and ageing 36: 122-127.

8. Mokaya J, Dotchin CL, Gray WK, et al. (2016) The accessibility of parkinson's disease medication in kenya: Results of a national survey. Mov Disord Clin Pract 3: 376-381.

9. Dotchin C, Walker R (2012) The management of Parkinson's disease in sub-saharan africa. Expert rev neurother 12: 661-666.

10. Fothergill-Misbah N, Maroo H, Hooker J, et al. (2020) Parkinson's disease medication in kenya-situation analysis. Pharmaceutical Journal of Kenya 24: 38-41.

11. Dotchin C, Jusabani A, Walker R (2011) Three year follow up of levodopa plus carbidopa treatment in a prevalent cohort of patients with Parkinson's disease in Hai, Tanzania. J neurol 258: 1649-1656.

12. Massano J, Bhatia KP (2012) Clinical approach to Parkinson's disease: Features, diagnosis, and principles of management. Cold Spring Harb Perspect Med 2: a008870.

13. Salawu FK, Danburam A, Olokoba AB (2010) Non-motor symptoms of parkinson's disease: Diagnosis and management. Niger J Med 19: 126-131.

14. Carter JH, Stewart BJ, Lyons KS, et al. (2008) Do motor and nonmotor symptoms in PD patients predict caregiver strain and depression? Movement Disord 23: 1211-1216. 
15. Ama de-Graft Aikins A, Unwin N, Agyemang C, et al. (2010) Tackling africa's chronic disease burden: From the local to the global. Global Health 6: 5.

16. Aboderin I (2004b) Decline in material family support for older people in urban Ghana, Africa: Understanding Processes and Causes of Change. J Gerontol B Psychol Sci Soc Sci 59: S128-S137.

17. Aboderin I (2006) Intergenerational support and old age in Africa: Transaction.

18. Gachuhi JKK (2005) Research capacity on ageing in Africa: Limitations and ways forward. British Society of Gerontology 15: 36-38.

19. Aboderin Isabella (2010) Global ageing: Perspectives from sub-Saharan Africa. In: Dannefer, Dale, Phillipson Chris, the SAGE Handbook of Social Gerontology. SAGE Publications, London, GB, 405-419.

20. Walga TK (2019) Understanding the experience and perspectives of parkinson's disease patients' caregivers. Rehabilitation Research and Practice 2019: 1-9.

21. De-Graft Aikins A, Boynton P, Atanga LL (2010) Developing effective chronic disease interventions in africa: Insights from ghana and cameroon. Global Health 6: 6.

22. Stacey M (1988) The sociology of health and healing. Unwin Hyman Ltd.

23. INDEPTH Network (2002) Population and health in developing countries. Population, health and survival at INDEPTH sites 1.

24. Dewhurst F, Dewhurst MJ, Gray WK, et al. (2012) The prevalence of neurological disorders in older people in Tanzania. Acta neurol Scand 127: 198-207.

25. Braun V, Clarke V (2006) Using thematic analysis in psychology. Qualitative Research in Psychology 3: 77-101.

26. Charmaz K (1983) Loss of self: A fundamental form of suffering in the chronically ill. Sociol Health IIIn 5: 168-195.

27. Bramley N, Eatough V (2005) The experience of living with Parkinson's disease: An interpretative phenomenological analysis case study. Psychology \& Health 20: 223-235.

28. Dotchin CL, Paddick SM, Longdon AR, et al. (2014) A comparison of caregiver burden in older persons and persons with Parkinson's disease or dementia in sub-Saharan Africa. Int psychogeriatr 26: 687-692.

29. Dotchin CL, Akinyemi RO, Gray WK, et al. (2013) Geriatric medicine: Services and training in Africa. Age Ageing 42: 124-128.

30. NICE (2017) Parkinson's disease in adults: NICE guideline.

31. Rosland A (2009) Sharing the Care: The Role of Family in Chronic Illness.

32. Soleimani MA, Bastani F, Negarandeh R, et al. (2016) Perceptions of people living with Parkinson's disease: A qualitative study in Iran. Br J Community Nurs 21: 188-195.

33. Chiong-Rivero H, Ryan GW, Flippen C, et al. (2011) Patients' and caregivers' experiences of the impact of Parkinson's disease on health status. Patient relat outcome meas 2011: 57-70.

34. Khlebtovsky A, Rigbi A, Melamed E, et al. (2012) Patient and caregiver perceptions of the social impact of advanced Parkinson's disease and dyskinesias. J Neural Transm 119: 1367-1371.
35. Rajiah K, Maharajan MK, Yeen SJ, et al. (2017) Quality of life and caregivers' burden of parkinson's disease. Neuroepidemiology 48: 131-137.

36. Soundy A, Stubbs B, Roskell C (2014) The experience of Parkinson's disease: A systematic review and meta-ethnography. Scientific World Journal 2014: 613592.

37. Apt $N$ (2002) Ageing and the changing role of the family and the community: An African Perspective. International Social Security Review 55: 39-47.

38. Apt N (2001) Rapid urbanization and living arrangements of older persons in Africa. UN Secretariat, Affairs EaS.

39. Aboderin I (2004a) Modernisation and ageing theory revisited: Current explanations of recent developing world and historical western shifts in material family support for older people. Ageing and Society. 24: 29-50.

40. Asare M, Danquah SA (2017) The African belief sytem and the patient's choice of treatment from existing health models: The case of ghana. Acta Psychopathologica 4: 49.

41. Mokgobi MG (2014) Understanding traditional African healing. Afr J Phys Health Educ Recreat Dance 20: 24-34.

42. Howson A (2009) Cultural Relativism. EBSCO Research Starters.

43. Omonzejele PF (2008) African concepts of health, disease, and treatment: An ethical inquiry. Explore (New York, NY) 4: 120-126.

44. Janzen JM (2017) Science in the moral space of health and healing paradigms in western equatorial Africa. In: Olsen WC, Sargent $\mathrm{C}$, African medical pluralism. Bloomington and Indianapolis. Indiana University Press 90-109.

45. Bingham V, Habermann B (2006) The influence of spirituality on family management of Parkinson's disease. J Neurosci Nurs 38: 422-427.

46. Janzen JM (1978) The quest for therapy in Lower Zaire. University of California Press.

47. Awah P (2006) Diabetes and traditional medicine in Africa. Diabetes Voice.

48. Chukwuneke FN, Ezeonu CT, Onyire BN, et al. (2012) Culture and biomedical care in Africa: The influence of culture on biomedical care in a traditional African society, Nigeria, West Africa. Niger J Med 21: 331-333.

49. Hampshire KR, Owusu SA (2013) Grandfathers, google, and dreams: Medical pluralism, globalization, and new healing encounters in ghana. Medical Anthropology 32: 247-265.

50. Sugishita K (2009) Traditional medicine, biomedicine and christianity in Modern Zambia. Journal of the International African Institute 79: 435-454.

51. Marsland R (2007) The modern traditional healer: Locating 'hybridity' in modern traditional medicine, southern Tanzania. Journal of Southern African Studies 33: 751-765.

52. McMillen H (2004) The adapting healer: Pioneering through shifting epidemiological and sociocultural landscapes. Soc Sci Med 59: 889-902.

53. Maffoni M, Giardini A, Pierobon A (2017) Stigma experienced by parkinson's disease patients: A descriptive review of qualitative studies 2017: 7203259.

DOI: $10.36959 / 459 / 606$

Copyright: (C) 2021 Fothergill-Misbah N, et al. This is an open-access article distributed under the terms of the Creative Commons Attribution License, which permits unrestricted use, distribution, and reproduction in any medium, provided the original author and source are credited.

SCHOLARS.DiRECT 